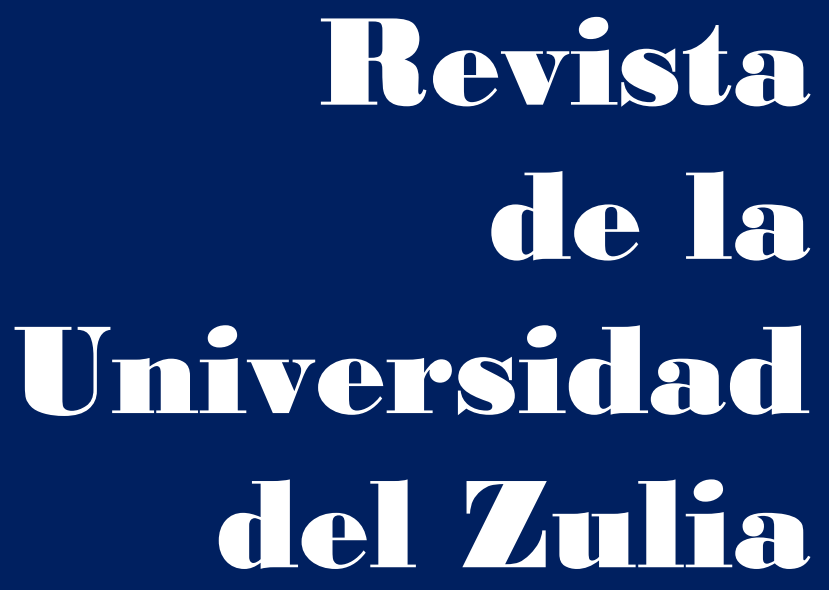

Fundada en 1947

por el Dr. Jesús Enrique Lossada

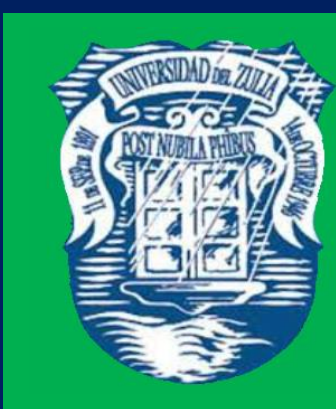

Ciencias del

Agred

Ingemieria

y Teenología
Añต 12 No 32

Enero - Abril 2021

Tercera Época

Maracailbo-Venezuela 


\title{
Variación estacional de testosterona fecal y desarrollo de astas en una subespecie tropical de venado cola blanca (Odocoileus virginianus) de la región Huasteca, México
}

\author{
Carlos Alberto Contreras Verteramo * \\ José Antonio Rangel Lucio ** \\ Sonia Antonieta Gallina Tessaro *** \\ Brenda Alvarado Sánchez ****
}

\section{RESUMEN}

Objetivo: analizar la relación entre el nivel de testosterona en heces fecales y el ciclo de las astas, así como determinar el período reproductivo del venado cola blanca (O. v. veraecrucis) en función del hábitat de cuatro Unidades de Manejo para la Conservación de Vida Silvestre (UMA) de la región Huasteca de Veracruz y San Luis Potosí, México. Método: El estudio se realizó en cuatro ranchos ganaderos registrados como UMA, ubicados en los estados de Veracruz y San Luis Potosí, en la región Huasteca de México. Se evaluaron veintisiete muestras fecales y ciento noventa y dos imágenes fotográficas de machos de venado cola blanca para identificar el patrón reproductivo anual. Las muestras e imágenes se recolectaron entre febrero de 2015 y julio de 2016. La concentración de testosterona en heces se determinó mediante pruebas ELISA. Se realizaron análisis estadísticos de regresión lineal y correlación para identificar la relación de la concentración de testosterona con el crecimiento promedio de las astas, de escalamiento multidimensional no métrico para la ordenación de los valores de concentración de testosterona observados, etapa de crecimiento de las astas y variables ambientales. Para agrupar los meses en que los animales presentan características reproductivas similares se aplicó un análisis de k-medias. Resultados: Fue posible identificar una relación positiva entre la variación anual de los niveles de testosterona y las etapas del ciclo de astas; las menores concentraciones hormonales ocurrieron de marzo a junio y coincidieron con la muda y crecimiento de astas nuevas, permitiendo identificar este lapso como no reproductivo, mientras que, entre julio y febrero el nivel de testosterona se mantuvo elevado, coincidiendo con la presencia de astas osificadas y libres de terciopelo, indicador de actividad reproductiva en los machos.

PALABRAS CLAVE: aprovechamiento sustentable; cérvidos; ciclo reproductivo; conservación; manejo de fauna.

* Instituto Tecnológico de Ciudad Victoria; Instituto Tecnológico Superior de Pánuco. E-mail: contrerc@itspanuco.edu.mx. ORCID: https://orcid.org/0000-0001-7848-9545

** Instituto Tecnológico de Ciudad Victoria. E-mail: antonio.rangel@itvictoria.mx. ORCID: https://orcid.org/0000-0002-4055-6527

*** Instituto de Ecología A. C. E-mail: sonia.gallina@inecol.mx. ORCID: https://orcid.org/00000002-8941-5186

**** Unidad Académica Multidisciplinaria Zona Huasteca Universidad Autónoma de San Luis Potosí. E-mail: balvarado@uaslp.mx. ORCID: https://orcid.org/0000-0002-6077-2665

Recibido: 11/09/2020

Aceptado: 30/10/2020 


\section{Seasonal changes of fecal testosterone and antler development in a tropical white-tailed deer (Odocoileus virginianus) subspecies of the Huasteca region, Mexico}

ABSTRACT

Objective: to analyze the relationship between the level of testosterone in feces and the antler cycle, as well as to determine the reproductive period of the white-tailed deer (O. v. Veraecrucis) based on the habitat of four Management Units for the Conservation of Vida Silvestre (UMA) from the Huasteca region of Veracruz and San Luis Potosí, Mexico. Method: The study was carried out in four cattle ranches registered as UMA, located in the states of Veracruz and San Luis Potosí, in the Huasteca region of Mexico. Twenty-seven fecal samples and one hundred and ninety-two photographic images of male white-tailed deer were evaluated to identify the annual reproductive pattern. The samples and images were collected between February 2015 and July 2016. Stool testosterone concentration was determined using ELISA tests. Statistical analyzes of linear regression and correlation were carried out to identify the relationship of the testosterone concentration with the average growth of the antlers, non-metric multidimensional scaling for the ordering of the observed testosterone concentration values, growth stage of the antlers and environmental variables. To group the months in which the animals present similar reproductive characteristics, a $\mathrm{k}$-means analysis was applied. Results: It was possible to identify a positive relationship between the annual variation of testosterone levels and the stages of the antler cycle; the lowest hormonal concentrations occurred from March to June and coincided with the molting and growth of new antlers, allowing to identify this period as non-reproductive, while, between July and February, the level of testosterone remained high, coinciding with the presence of ossified antlers. and velvet free, an indicator of reproductive activity in males.

KEY WORDS: sustainable use; cervids; reproductive cycle; conservation; wildlife management.

\section{Introducción}

Entre las especies de cérvidos que se distribuyen en México, el venado cola blanca (Odocoileus virginianus Zimmermann, 1780), es el más importante desde la perspectiva de aprovechamiento cinegético (Clemente et al. 2015). Esta especie se distribuye en la mayor parte del territorio mexicano exceptuando la península de Baja California, con registros de densidad poblacional entre 0.37 y 28.0 ind $/ \mathrm{km}^{2}$; estas características contribuyen al valor 
REVISTA DE LA UNIVERSIDAD DEL ZULIA. 3ª época. Año 12 Nº 32, 2021

Carlos Alberto Contreras et al. ///Variación estacional de testosterona fecal y desarrollo de astas... 7-23

DOI: http://dx.doi.org/10.46925//rdluz.32.02

ecológico, económico y cultural de la especie (Halls 1984; Gallina y López 2008; Beltrán y Díaz de la Vega 2010; Gallina et al. 2010; Piña y Trejo 2014).

A partir de la creación del sistema de Unidades de Manejo para la Conservación de la Vida Silvestre (UMA) en 1997, para aprovechar especies de fauna como el venado cola blanca, es necesario desarrollar un plan de manejo con información detallada sobre sus características biológicas y hábitat (Secretaría de Medio Ambiente y Recursos Naturales SEMARNAT 2016). Para que el manejo de fauna silvestre sea viable, temporal y espacialmente, sin efectos negativos en la biodiversidad y productividad regional, es fundamental conocer aspectos de la especie objetivo, tales como requerimiento de espacio, movimientos estacionales, dinámica poblacional, alimentación y ciclo reproductivo (Rojo et al. 2007; Villarreal 2013; Waber et al. 2013).

En México, la mayoría de las subespecies de venado cola blanca se distribuye en regiones tropicales, aunque sujetas a alguna modalidad de aprovechamiento; situación que obliga al desarrollo de planes de manejo específicos para cada eco-región. Esta necesidad también obliga a prestar atención a la deficiencia de conocimiento biológico de venados en zonas tropicales, como el patrón reproductivo, uso de hábitat y dieta, entre otros (Vaughan 1994; Gallina y Mandujano 2009; Vishnu-Savanth y Saseendran 2012).

Los criterios para manejar y aprovechar de forma sustentable el venado cola blanca se han generalizado para México; sin embargo, para diversas zonas tropicales, incluida la región Huasteca, aún falta documentar elementos básicos de la biología de la especie, como alimentación o ciclo reproductivo que deriven en planes de manejo adecuados para su aprovechamiento sustentable (Rojo et al. 2007; Mandujano 2011; Villarreal 2013).

Las astas son una característica importante de los machos (y que le da valor a la especie desde el punto de vista cinegético) que permite conocer el grado de salud de las poblaciones debido a que su desarrollo se relaciona con la calidad del hábitat, con la ingesta de nutrimentos y edad de los animales. Una de las metas iniciales de los programas de manejo de venados es el incremento del tamaño de astas durante la vida de los animales (Brothers y Ray 1975; Miller y Marchinton 1995; Demarais y Strickland 2011).

Los venados presentan actividad reproductiva intermitente (ciclo anual), sólo restringida por el período de estro en hembras, que regula la liberación de óvulos, y producción de esperma para lograr el éxito. Este proceso es afectado por factores ambientales 
REVISTA DE LA UNIVERSIDAD DEL ZULIA. 3ª época. Año 12 Nº 32, 2021 Carlos Alberto Contreras et al. ///Variación estacional de testosterona fecal y desarrollo de astas... 7-23 DOI: http://dx.doi.org/10.46925//rdluz.32.02

como precipitación pluvial y temperatura, además de la disponibilidad de alimento; sin embargo, el fotoperíodo es más importante para definir la época reproductiva (Goss 1983; Jacobson y Waldhalm 1992; Hansen 2011; Glover 2012; Vega et al. 2020).

El período de tiempo que los cérvidos mantienen las astas limpias de terciopelo está correlacionado de forma positiva con la concentración de testosterona en plasma, la cual se incrementa en la época reproductiva. En venados de zonas boreales, el aumento y disminución de la concentración de testosterona en plasma se presenta repentina e intensamente, ocasionando mineralización de astas, caída de terciopelo e incremento en espermatogénesis; las astas se conservan por un período de tiempo corto y la producción de esperma se suspende con la muda. En cambio, en regiones ecuatoriales la testosterona se incrementa y decrece lentamente, sin alcanzar niveles que detengan la espermatogénesis; las astas y la actividad sexual se mantienen por un periodo mayor en el año (Loudon y Curlewis 1988; Bubenik 1990; Pelletier et al. 2003; Newbolt et al. 2017; Stewart et al. 2018; Weerasekera et al. 2020).

Los glucocorticoides en heces fecales indican el nivel de secreción de ciertas hormonas por un período de tiempo, ofrecen un panorama del nivel hormonal en muestras de plasma o fecales y son una alternativa para estudios de reproducción en cérvidos y otras especies, ya que la manipulación requerida de los individuos es mínima o nula (Pelletier et al. 2003; Mc. Coy y Ditchkoff 2012; Arroyo et al. 2013; López-Cobá y Montes-Pérez 2016).

Conocer la temporalidad del ciclo reproductivo para el venado cola blanca de la región Huasteca, hará posible la realización de estudios de otra índole, como los nutricionales, y relacionarlos con la variación fisiológica de la especie a lo largo del año.

El objetivo de esta investigación fue determinar la relación entre el nivel de testosterona en heces fecales y el ciclo de las astas, e identificar el período reproductivo del venado cola blanca (Odocoileus virginianus veraecrucis), en función del hábitat de cuatro UMA de la región Huasteca de Veracruz y San Luis Potosí, México. La hipótesis del estudio indica que el nivel de testosterona fecal será mayor durante el lapso en que los venados presentan astas osificadas, libres de terciopelo y será coincidente con el período reproductivo, mientras que el nivel menor se presentará cuando los animales mudan las astas e inicia el crecimiento de una nueva cornamenta. 


\section{Materiales y métodos}

\section{1. Área de estudio}

El estudio fue realizado en cuatro ranchos ganaderos de la región Huasteca de México, registrados como Unidades de Manejo para la Conservación de la Vida Silvestre (UMA); dos

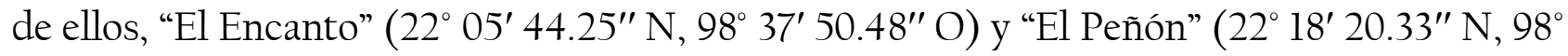
52' 54.25" O) están situados en el municipio de Tamuín, San Luis Potosí, mientras que "El

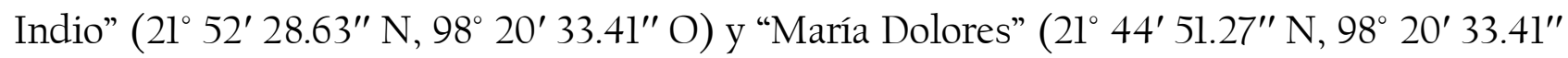
O) se ubican en el municipio de Pánuco, Veracruz.

El clima de la región se clasifica como cálido subhúmedo con lluvias en verano (Aw); la mayor parte de precipitación pluvial (81.6 \%) ocurre entre junio y octubre, mientras que la época de sequía de febrero a mayo. Los valores máximos de temperatura se registran entre mayo y septiembre $\left(37-45^{\circ} \mathrm{C}\right.$ ), la temporada fría entre diciembre y febrero (García 2004; Centro de Investigación Científica y de Educación Superior de Ensenada CICESE 2015; Instituto Nacional de Investigaciones Forestales, Agrícolas y Pecuarias INIFAP 2018). La principal actividad productiva desarrollada en estos predios es la ganadería extensiva de bovinos, con cargas que van de 1.95 a 2.3 unidades animales por hectárea (UA/ha) (Comisión Técnico Consultiva para la Determinación de Coeficientes de Agostadero COTECOCA, 2002). En los ranchos también se realiza agricultura de temporal en pequeña escala, para obtención de especies forrajeras y como actividad económica alternativa; asimismo, el venado cola blanca se maneja y aprovecha mediante cacería deportiva, conforme a la normatividad vigente.

La vegetación característica de los sitios de muestreo incluye pastizales inducidos y especies nativas típicas de selvas bajas caducifolias y subcaducifolias espinosas como chijol (Piscidia communis), guásima (Guazuma ulmifolia), ébano (Ebenopsis ebano), huizache (Acacia farnesiana), gavia (A. pringlei), cornezuelo (A. cornigera), uña de gato (A. gregii), limoncillo (Xanthoxylum fagara), pusgual (Croton cortesianus), palma real (Sabal mexicana), cerón (Phyllostilon brasiliense), chaca (Bursera simaruba) y mezquite (Prosopis glandulosa), entre otras (Márquez y Márquez 2009; Ellis y Martínez 2010; Comisión Nacional de Áreas Naturales Protegidas CONANP - SEMARNAT 2014). 
REVISTA DE LA UNIVERSIDAD DEL ZULIA. 3ª época. Año 12 Nº 32, 2021 Carlos Alberto Contreras et al. ///Variación estacional de testosterona fecal y desarrollo de astas... 7-23 DOI: http://dx.doi.org/10.46925//rdluz.32.02

En la región se han registrado especies de mamíferos silvestres medianos y grandes como coatí (Nasua narica), mapache (Procyon lotor), zorrillo rayado (Conepatus leuconotus), armadillo (Dasypus novemcinctus), tlacuache (Didelphis virginiana), conejo (Sylvilagus floridanus), ardilla (Sciurus aureogaster), pecarí de collar (Pecari tajacu), coyote (Canis latrans), zorra gris (Urocyon cinereoargenteus), jaguarundi (Puma yagouaroundi), tigrillo (Leopardus wiedii), ocelote (Leopardus pardalis); aves como guajolote silvestre (Meleagris gallopavo), loro tamaulipeco (Amazona viridigenalis), chachalaca (Ortalis vetula), paloma morada (Columba flavirostris), paloma arroyera (Leptotila verreauxi), paloma huilota (Zenaida macroura), codorniz común (Colinus virginianus), pájaros carpinteros (Melanerpes aurifrons y Picoides scalaris) y diversas especies de colibríes y aves canoras (Ellis y Martínez 2010; CONANP - SEMARNAT 2014).

También se han observado gato montés (Lynx rufus) y loro cabeza amarilla (Amazona oratrix) además de diversas especies de reptiles, entre las que destacan cocodrilo de pantano (Crocodylus moreletii), iguana negra (Ctenosaura acanthura), serpiente de cascabel (Crotalus durissus), mazacuate (Boa constrictor), serpiente coral (Micrurus browni), cantil (Agkistrodon taylori), cincuate (Pituophis sp.) y nauyaca (Bothrops asper), entre otros.

\subsection{Colecta de muestras}

Entre febrero de 2015 y julio de 2016 se colectaron muestras fecales de 27 ejemplares machos de venado cola blanca; las excretas fueron tomadas del recto de venados cobrados mediante caza deportiva durante las temporadas 2014-2015 y 2015-2016 extendidas al resto del año por autorización de colecta científica SGPA/DGVS/03414/15; tres muestras fecales se tomaron en la UMA el Indio (DGVS-CR-EX-3291-VER), cuatro en María Dolores (SEMARNAT-UMA-EX-0352-VER/14), ocho en El Encanto (DGVS-CR-EX-2069-SLP) y 12 en El Peñón (DGVS-UMA-EX-3670-SLP). Las excretas fueron colocadas en tubos de $50 \mathrm{~mL}$ para centrifugado, dentro de una hielera con refrigerante para evitar su degradación, trasladadas al laboratorio de ciencias biomédicas de la Unidad Académica Multidisciplinaria Zona Huasteca de la Universidad Autónoma de San Luis Potosí en Cd. Valles, S. L. P. y conservadas en congelación a $23^{\circ} \mathrm{C}$ bajo cero hasta su análisis (Millspaugh y Washburn 2004; Barja et al. 2006; Yang et al. 2010). 
REVISTA DE LA UNIVERSIDAD DEL ZULIA. 3ª época. Año 12 Nº 32, 2021 Carlos Alberto Contreras et al. ///Variación estacional de testosterona fecal y desarrollo de astas... 7-23 DOI: http://dx.doi.org/10.46925//rdluz.32.02

Se adquirió la información de precipitación pluvial mensual y media mensual histórica, así como temperaturas media, mínima y máxima diarias, registradas por la estación meteorológica 36726 INIFAP-Ébano, San Luis Potosí, durante 2015 y 2016 (CICESE 2015; INIFAP 2018). Con apoyo de la calculadora solar de la Administración Nacional Oceánica y Atmosférica (NOAA), se realizó el cálculo de las horas/luz, o foto período, de cada día del año a los $22^{\circ}$ latitud Norte y $98^{\circ}$ longitud Oeste, para integrar un promedio mensual (NOAA 2018).

\subsection{Análisis de muestras}

El análisis de muestras fecales para cuantificar el contenido de testosterona se efectuó en el laboratorio de Ciencias Biomédicas de la Unidad Académica Interdisciplinaria Zona Huasteca, de la Universidad Autónoma de San Luis Potosí.

Paralelamente, se analizaron 192 imágenes fotográficas de venados captados mediante cuatro cámaras trampa, entre agosto de 2015 y julio de 2016. En las dos UMA del municipio de Tamuín, S.L.P. fueron capturadas 103 imágenes con dos cámaras, mientras que, en las dos UMA del municipio de Pánuco, Veracruz se captaron 86 imágenes de venados. Con estas imágenes se clasificó la etapa del ciclo de las astas de cada individuo, asignándoles un valor (Tabla 1). Asimismo, las imágenes se ordenaron de acuerdo con el mes de captura y se calculó el promedio mensual de desarrollo de astas. También se registraron los meses con presencia de cervatos, directamente de forma personal o por empleados de las UMA e indirectamente al ser captados por cámaras trampa.

La medición de testosterona se realizó por triplicado mediante análisis ELISA (Enzymelinked immunosorbent assay) de tipo competitivo, se empleó un kit comercial Testosterone ELISA Kit de la marca Cayman Chemical y se siguieron las instrucciones del fabricante (Cayman Chemical 2017). Este ensayo se basa en la competencia que ocurre entre la testosterona de la muestra y la testosterona HRP conjugada por una constante unión de anticuerpos de conejo anti-testosterona (Tracer), por una cantidad limitada del antisuero de testosterona. La concentración del Tracer de testosterona es constante mientras que la concentración de testosterona de la muestra es variable. Por lo tanto, la cantidad del Tracer de testosterona capaz de unirse al antisuero de testosterona es inversamente proporcional a la concentración de testosterona de la muestra. (Munro y Stabenfeldt 1984; Guzmán-Vásquez 2004). 
REVISTA DE LA UNIVERSIDAD DEL ZULIA. 3ª época. Año 12 Nº 32, 2021

Carlos Alberto Contreras et al. ///Variación estacional de testosterona fecal y desarrollo de astas... 7-23

DOI: http://dx.doi.org/10.46925//rdluz.32.02

Los resultados de la concentración de testosterona en heces fecales se sometieron a un análisis de correlación lineal con el crecimiento promedio de las astas. Además, para ordenar los datos, extraer el gradiente dominante de variación y visualizar la distancia entre ellos en un espacio de dos dimensiones, se aplicó un análisis de escalamiento multidimensional no métrico (EMNM) a los valores de concentración de testosterona observados, etapa de crecimiento de las astas y variables ambientales (McGarigal et al. 2000; Greenacre y Primicerio 2013).

Con el fin de agrupar los meses con características similares e identificar las variables que determinaron dicha aglomeración, se aplicó un análisis de agrupación de K-medias, con las mismas variables analizadas en EMNM (MacQueen 1967; Greenacre y Primicerio 2013; Hair et al. 2014; XLSTAT 2017), a las clases resultantes se les aplicó una prueba t-Student para comparar los niveles de testosterona fecal.

\section{Resultados}

El análisis de heces fecales indica que, desde julio hasta febrero, se presenta el nivel máximo de testosterona (Tabla 2). Por otro lado, las imágenes fotográficas muestran que, en ese mismo período de tiempo, las cornamentas de los venados se encuentran osificadas y limpias (sin terciopelo); en contraste, la menor concentración de testosterona se detectó entre marzo y junio, temporada en que los venados mudan las astas e inician un nuevo ciclo de crecimiento (Tabla 2).

La correlación entre concentración de testosterona en heces fecales y la etapa de desarrollo del mismo período fue positiva y explicó $78 \%$ de la variación de los datos $(\mathrm{R}=$ $0.885 ; \mathrm{R}^{2}=0.782 ; \mathrm{p}<0.0001$ ).

Los resultados del análisis de escalamiento multidimensional no métrico (NMMDS), muestran que los meses de julio a febrero se encuentran cercanos en el espacio canónico, esto sugiere que, durante ese lapso, en el área de estudio, los venados presentan características similares en cuanto a concentración de testosterona en heces y desarrollo de astas; de igual forma, es posible intuir que entre marzo y mayo los valores de testosterona fecal y desarrollo de astas son comunes. En contraste, junio se encuentra en una posición intermedia entre los dos grupos, indicando probablemente que en este período se presenta la transición entre el estado no reproductivo y reproductivo (Stress de Kruskal = 0.049) (Figura 1). 
REVISTA DE LA UNIVERSIDAD DEL ZULIA. 3ª época. Año 12 Nº 32, 2021

Carlos Alberto Contreras et al. ///Variación estacional de testosterona fecal y desarrollo de astas... 7-23

DOI: http://dx.doi.org/10.46925//rdluz.32.02

Mediante el análisis de K-medias se aglutinaron los meses del año en dos grupos. El primero, denominado período reproductivo, incluye los meses de julio a febrero y el segundo o no reproductivo, de marzo a junio. Las variables que determinaron este patrón de agrupación fueron concentración de testosterona ( $\mathrm{F}=89.59 ; \mathrm{P}<0.0001)$ y etapa de desarrollo de astas $(\mathrm{F}=60.21 ; \mathrm{P}<0.0001)$ (Tabla 3).

Adicionalmente, la aplicación de la prueba t-Student, confirmó que los machos de venado cola blanca en la región Huasteca presentan un período fértil o reproductivo desde julio hasta febrero, debido a que los niveles de testosterona fecal permanecen altos y mantienen astas osificadas $(\mathrm{t}=10.08 ; \mathrm{P}<0.0001)$, así como un período no reproductivo entre marzo y junio en los que se puede observar una disminución de concentración de la hormona (Figura 3).

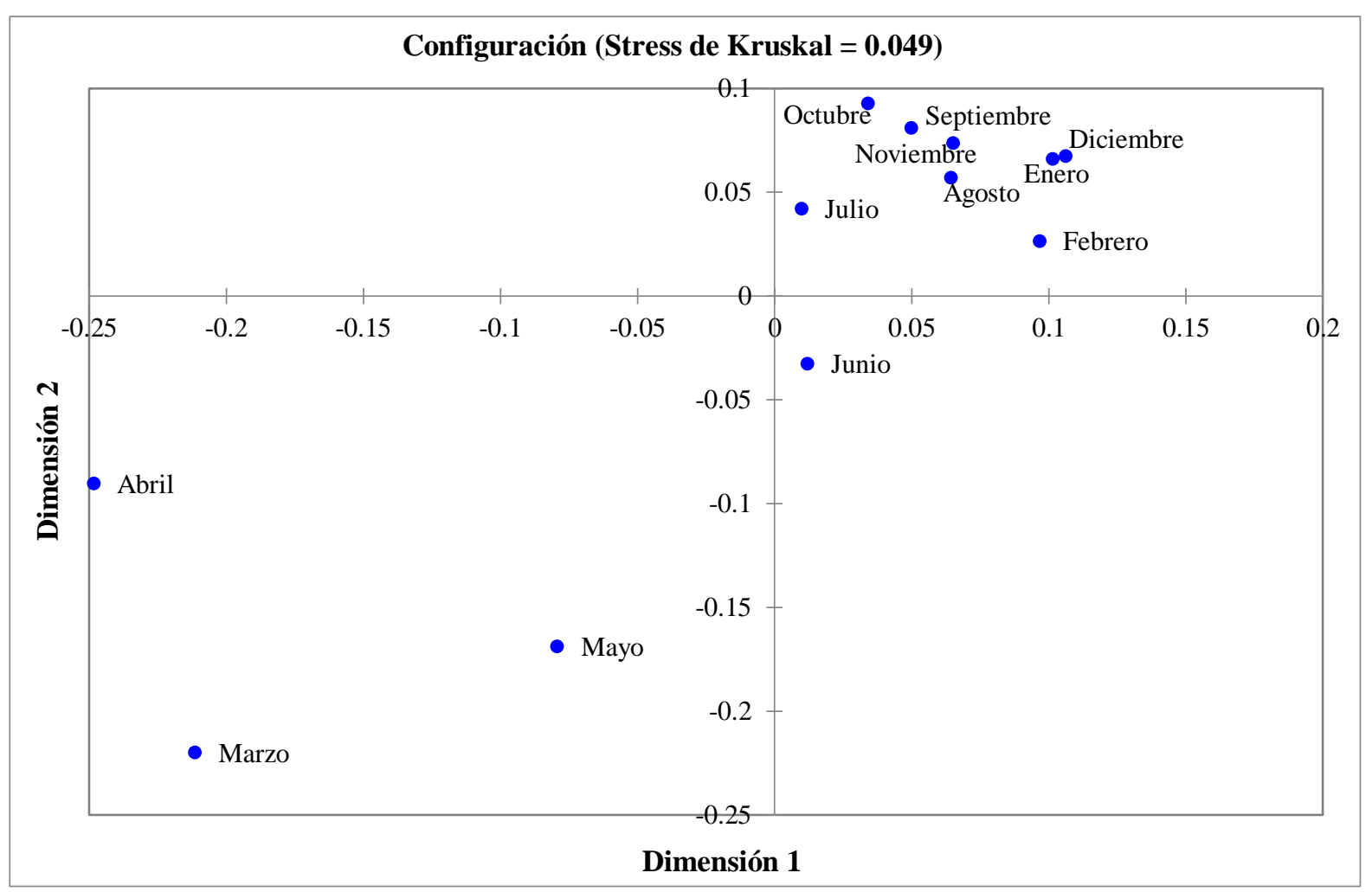

Figura 1. Distancias canónicas entre meses, de acuerdo con la ordenación por NMMDS utilizando datos de concentración de testosterona y desarrollo de astas en machos de venado cola blanca, así como variables climáticas en la zona Huasteca de México. 
REVISTA DE LA UNIVERSIDAD DEL ZULIA. 3ª época. Año 12 Nº 32, 2021

Carlos Alberto Contreras et al. ///Variación estacional de testosterona fecal y desarrollo de astas... 7-23

DOI: http://dx.doi.org/10.46925//rdluz.32.02

Etapa de desarrollo de las astas
Sin astas $\begin{array}{cc}\text { Crecimiento } & \text { Crecimiento } \\ \text { inicial } & \text { medio }\end{array}$

\section{Crecimiento}

completo

con

terciopelo

Valor asignado

1

2

3

4

5

Imagen de la asta.

(McMillin et al. 1974).
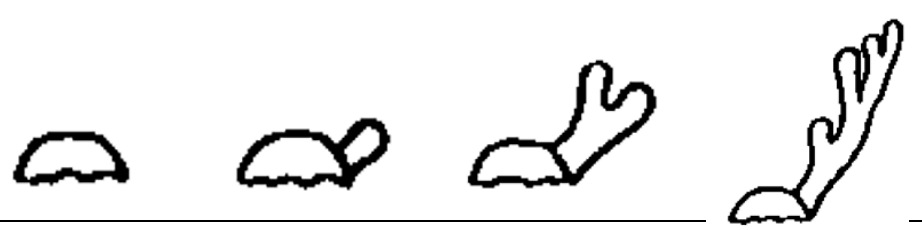

Astas

limpias

Tabla 1. Etapas de desarrollo de las astas de venado cola blanca en la zona Huasteca de México, registradas fotográficamente entre agosto de 2015 y julio de 2016 y adaptadas a las imágenes propuestas por McMillin et al. (1974).

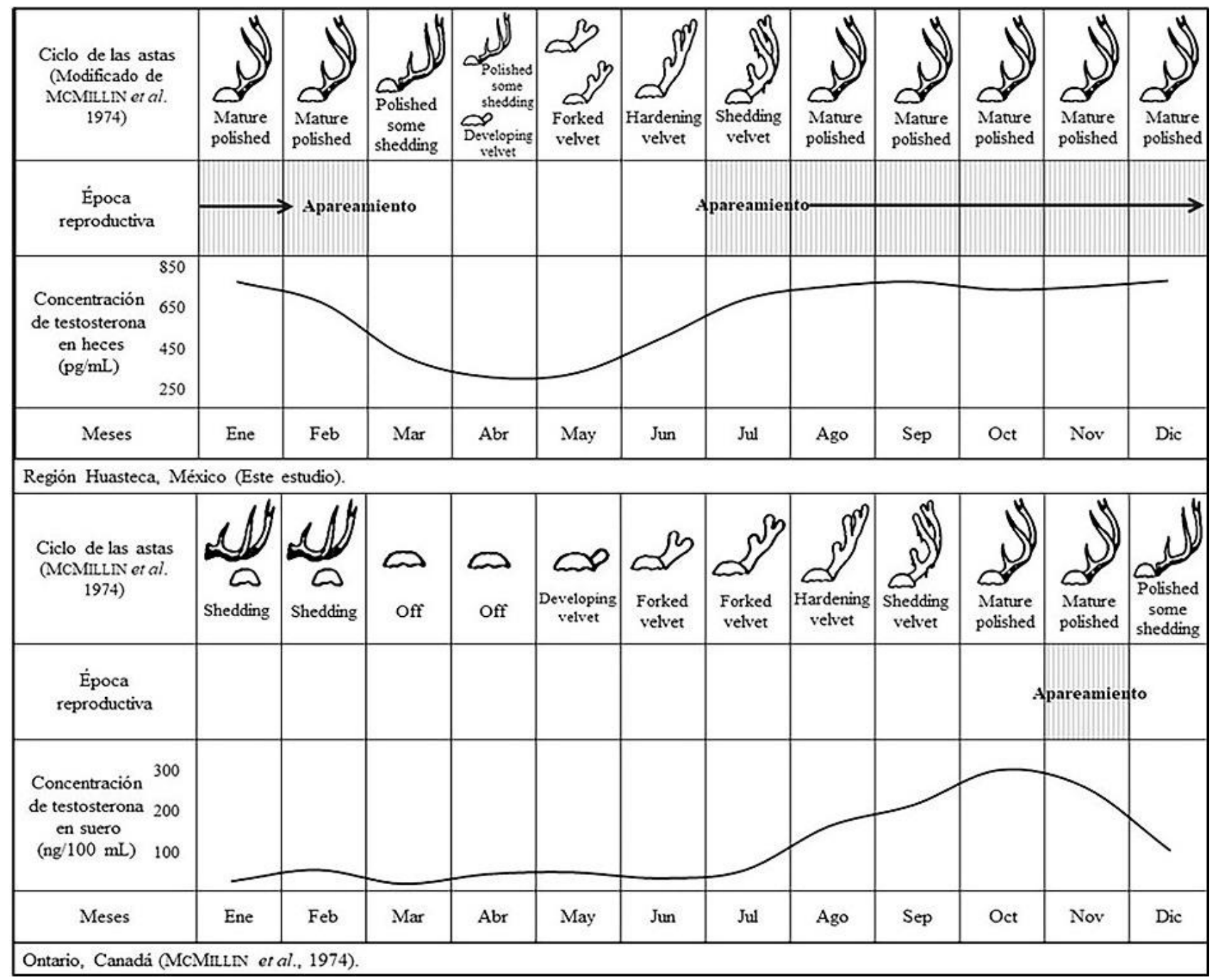

Figura 2. Comparación de características del ciclo de astas y concentración de testosterona en machos de venado cola blanca en la región Huasteca de México (este estudio) y en Ontario, Canadá (McMillin et al., 1974). 
REVISTA DE LA UNIVERSIDAD DEL ZULIA. 3ª época. Año 12 Nº 32, 2021

Carlos Alberto Contreras et al. ///Variación estacional de testosterona fecal y desarrollo de astas... 7-23

DOI: http://dx.doi.org/10.46925//rdluz.32.02

\section{Discusión y conclusiones}

Estos resultados contrastan de manera importante con estudios similares realizados en el Norte de México, donde los venados ostentan astas limpias de octubre a febrero, pero las diferencias son de mayor magnitud hacia latitudes septentrionales, donde el período de mayor concentración de testosterona ocurre entre octubre y diciembre (McMillin et al. 1974; Miriarchi et al. 1978; Bubenik et al. 1990, 2005; Villarreal 1999; Vega et al. 2020). Los resultados de este estudio también difieren de aquellos en los que se ha identificado la ausencia de una periodicidad reproductiva característica de latitudes ecuatoriales, hasta aproximadamente $15^{\circ}$ de latitud (Goss 1983; Halls 1984).

\begin{tabular}{lcccc}
\hline \multicolumn{1}{c}{ Mes } & $\begin{array}{c}\text { Testosterona } \\
(\mathrm{pg} / \mathrm{mL})\end{array}$ & $\begin{array}{c}\text { Muestras } \\
(\mathrm{n}=27)\end{array}$ & $\begin{array}{c}\text { Etapa de } \\
\text { desarrollo de } \\
\text { astas (moda) }\end{array}$ & $\begin{array}{c}\text { Fotografías } \\
(\mathrm{n}=192)\end{array}$ \\
\hline Enero & 745.6 & 5 & 5 & 19 \\
Febrero & 706.3 & 4 & 5 & 16 \\
Marzo & 524.7 & 2 & 2 & 23 \\
Abril & 385.5 & 3 & 3 & 27 \\
Mayo & 382.9 & 3 & 3 & 19 \\
Junio & 515.5 & 2 & 4 & 12 \\
Julio & 724 & 2 & 5 & 15 \\
Agosto & 716.7 & 2 & 5 & 11 \\
Septiembre & 761.3 & 2 & 5 & 8 \\
Octubre & 724.9 & 3 & 5 & 10 \\
Noviembre & 735.5 & 1 & 5 & 15 \\
Diciembre & 753.2 & 2 & 5 & 10 \\
\hline
\end{tabular}

Tabla 2. Concentración mensual de testosterona en heces y etapas de desarrollo de astas de venado cola blanca en la zona Huasteca de México. Período: enero 2015 a febrero 2016. 
REVISTA DE LA UNIVERSIDAD DEL ZULIA. 3ª época. Año 12 Nº 32, 2021

Carlos Alberto Contreras et al. ///Variación estacional de testosterona fecal y desarrollo de astas... 7-23

DOI: http://dx.doi.org/10.46925//rdluz.32.02

\begin{tabular}{lll}
\hline \multicolumn{1}{c}{ Grupo } & \multicolumn{1}{c}{1} & \multicolumn{1}{c}{2} \\
\hline Varianza intraclase & 14032.195 & 12968.486 \\
Distancia mínima al centroide & 54.143 & 33.17 \\
Distancia media al centroide & 106.039 & 86.552 \\
\hline & Julio & Marzo \\
& Agosto & Abril \\
Meses que integran los grupos & Septiembre & Mayo \\
& Octubre & Junio \\
& Noviembre & \\
& Diciembre & \\
& Enero & \\
\hline
\end{tabular}

Tabla 3. Resultado del análisis de K-medias que muestra los meses que integran los grupos reproductivo (1) y no reproductivo (2) así como sus características.

La diferencia en la concentración media de testosterona entre épocas no reproductiva y reproductiva, observada en este estudio es similar a la registrada por Valdespino y Martínez-Romero (2014) en el Estado de Puebla y, aunque no tan amplia como la identificada por McCoy y Ditchkoff (2012) en Alabama, Estados Unidos, permite observar un patrón similar en el comportamiento reproductivo y ciclo de las astas de los venados.

El nivel de testosterona en heces fecales de venados cola blanca machos fue máximo entre julio y febrero, y presentaron astas osificadas limpias de terciopelo. Por el contrario, los niveles de testosterona disminuyen entre marzo y junio, período en que ocurre la muda, crecimiento y osificación de un nuevo par de astas.

En el caso de los venados, la temporalidad del aprovechamiento cinegético debe coincidir con el período reproductivo, de modo que los animales posean características que los hagan atractivos a los cazadores, como astas completamente desarrolladas y cuello engrosado; además, durante ese período su atención se enfoca en la búsqueda de hembras e incrementa su actividad diurna, pero se expone más y facilita su caza.

En la región Huasteca, la disminución de horas luz subsecuente al solsticio de verano y el aumento en la disponibilidad de alimento ocasionado por la temporada de lluvias, aparentemente son factores que influyen en el incremento del nivel de testosterona e inicio de la época de reproducción del venado cola blanca. En contraparte, el menor nivel de 
REVISTA DE LA UNIVERSIDAD DEL ZULIA. 3ª época. Año 12 Nº 32, 2021

Carlos Alberto Contreras et al. ///Variación estacional de testosterona fecal y desarrollo de astas... 7-23

DOI: http://dx.doi.org/10.46925//rdluz.32.02

testosterona se observa cuando aumenta el fotoperíodo y disminuye la cantidad de alimento, durante la época de sequía al final del invierno e inicio de la primavera.

Los resultados permiten identificar al periodo comprendido por los meses de septiembre octubre y noviembre, es decir la porción intermedia de la época de reproducción, como el más adecuado para el aprovechamiento cinegético en la región Huasteca, de esta manera se minimiza el efecto de eliminar de la población a los machos con mejores características de trofeo antes de haberse reproducido.

La metodología propuesta en este trabajo es susceptible de empleo en forma directa como herramienta para definir los períodos de aprovechamiento en distintas regiones del país y con algunas variaciones para identificar características poblacionales como porcentajes de preñez, relación de sexos o para identificar el comportamiento y función de las hormonas masculinas en hembras y viceversa.

\section{Referencias}

Arroyo, R. E., G. C. Vital y M. P. Lavín. (2013). Análisis fecales en el estudio de la reproducción en cérvidos y su papel en la conservación. Bio Ciencias 2:130-139.

Barja, I., G. Silván, J. Illera, S. Rosellini y A. Piñeiro (2006). La cuantificación de hormonas esteroides sexuales en heces de lobo ibérico (Canis lupus signatus): un método no invasivo de sexado como alternativa a los análisis moleculares. Oppidum 2:363-380.

Beltrán, V. y A. Díaz de la Vega (2010). Estimación de la densidad poblacional del venado cola blanca texano (Odocoileus virginianus texanus), introducido en la UMA "Ejido de Amanalco" Estado de México. Ciencia ergo-sum 17:154-158.

Brothers, A. y M. E. Ray (1975). Producing quality whitetails. Caesar Kleberg Wildl. Res. Inst., Kingsville, Tx. U.S.A. 246 p.

Bubenik, A. B. (1990). Evolution of Horns, Pronghorns, and Antlers. Pp: 3-113.

in Horns, pronghorns, and antlers. Evolution, morphology, physiology, and social significance (Bubenik G. A. y A. B. Bubenik, eds.). Springer-Verlag. New York, U.S.A.

Bubenik, G. A., R. D. Brown y D. Schams (1990). The effect of latitude on the seasonal pattern of reproductive hormones in the male white-tailed deer. Comparative Biochemistry y Physiology 97A:253-257.

Bubenik, G. A., K. V. Miller, A. L. Lister, D. A. Osborn, L. Bartos y G. Van Der Kraak (2005). Testosterone and estradiol concentrations in serum, velvet skin, and growing antler bone of male white-tailed deer. Journal of Experimental Zoology 303A:186-192. 
Cayman Chemical (2017). Testosterone Elisa kit, Item No. 582701. Cayman Chemical Company. Ann Arbor, MI, U.S.A. 31 p.

Centro de Investigación Científica y de Educación Superior de Ensenada (CICESE). (2015). Base de datos climatológica nacional (Sistema CLICOM) http://clicom-mex.cicese.mx/ (consultada el 4 de marzo de 2019).

Clemente, F., V. Cessa, C. Cortez, L. Tarango and P. Arenas (2015). Commercial extenders and freezing curves for the preservation of sperm cells of white-tailed deer (Odocoileus virginianus). Journal of Applied Animal Research 43:468-473. https://doi.org/10.1080/09712119.2014.980422

CONANP (Comisión Nacional de Áreas Naturales Protegidas) - SEMARNAT (Secretaría de Medio Ambiente y Recursos Naturales). 2014. Programa de Manejo Reserva de la Biosfera Sierra del Abra Tanchipa. Comisión Nacional de Áreas Naturales Protegidas, Secretaría de Medio Ambiente y Recursos Naturales. México. pp:134 - 196.

COTECOCA (Comisión Técnico Consultiva de Coeficientes de Agostadero). 2002. Coeficientes de agostadero por entidad federativa. Comisión Técnico Consultiva de Coeficientes de Agostadero SAGARPA - México. http://www.paot.org.mx/centro/inesemarnat/informe02/estadisticas_2000/compendio_2000/02dim_economica/02_02_Agricult ura/data_agricultura/CuadroII.2.4.3a.htm (Consulta: 18/01/2019)

Demarais, S. y B. Strickland. (2011). Antlers. in Biology and management of white-tailed deer. Hewitt, D. G. CRC Press. E.U.A. pp: 107-146.

Ellis, E. A. y M. Martínez (2010). Vegetación y uso de suelo. in Atlas del patrimonio natural histórico y cultural de Veracruz. (Florescano E. y J. Ortiz, eds) Comisión del Estado de Veracruz para la Conmemoración de la Independencia Nacional y de la Revolución Mexicana. Xalapa, Veracruz, México. pp:205-218.

Gallina, S. y A. H. López (2008). Odocoileus virginianus. The IUCN Red List of Threatened Species. Version 2014.1. www.iucnredlist.org.

Gallina, S., y S. Mandujano (2009). Research on ecology, conservation and management of wild ungulates in Mexico. Tropical Conservation Science 2:116-117.

Gallina, S., S. Mandujano, J. Bello, H. López-Fernández y M. Weber (2010). White-tailed deer Odocoileus virginianus (Zimmermann, 1780). 101-118 pp in Neotropical Cervidology: Biology and medicine of Latin American deer (Duarte, J. M. B., y S. González, S. eds.) Jaboticabal, Brazil: Funep and Gland, Switzerland, IUCN.

García, E. (2004). Modificaciones al sistema de clasificación climática de Koppen (5ª ed). Serie Libros, núm. 6, Instituto de Geografía, UNAM. México.

Glover, T. (2012). Mating Males. An evolutionary perspective on mammalian reproduction. Cambridge University Press. United Kingdom. 202 p. 
REVISTA DE LA UNIVERSIDAD DEL ZULIA. 3ª época. Año 12 Nº 32, 2021 Carlos Alberto Contreras et al. ///Variación estacional de testosterona fecal y desarrollo de astas... 7-23 DOI: http://dx.doi.org/10.46925//rdluz.32.02

Goss, R. J. (1983). Deer Antlers: Regeneration, Function, and Evolution. Academic Press. New York, E.U.A.

Greenacre, M y R. Primicerio. (2013). Multivariate analysis of ecological data. Fundación BBVA, Bilbao, Spain. 331 p.

Guzmán-Vásquez, E. (2004). Las pruebas de Elisa. Gaceta Médica Mexicana 140:48-49.

Hair, J., W. Black, W. Babin y R. Anderson (2014). Multivariate data analysis. 7a Ed. Pearson Education Ltd. London, UK. 761 p.

Halls, L. K. (1984). White-tailed deer. Ecology and management. Stackpole books. Harrisburg, E.U.A. 870 p.

Hansen, L. (2011). Extensive management. Pp: 413-440 in Biology and management of whitetailed deer. (Hewitt, D. ed.) CRC Press, Boca Raton, USA.

INIFAP (Instituto Nacional de Investigaciones Forestales, Agrícolas y Pecuarias). 2018. Laboratorio Nacional de Modelaje y Sensores Remotos, Instituto Nacional de Investigaciones Forestales, Agrícolas y Pecuarias. México. Consultado el 21/05/2018 en: http://clima.inifap.gob.mx/lnmysr/Estaciones/ConsultaDiariosl5Min?Estado=23yEstacion=3 6726

Jacobson, H. A. y S. J. Waldhalm. (1992). Antler cycles of a white-tailed deer with congenital anophthalmia. Pp: 520-524 in The Biology of Deer. (Brown, R. D. ed.) Springer-Verlag, New York, E.U.A.

López-Cobá, E. H. y R.C. Montes-Pérez (2016). Valoración de metabolitos de testosterona, progesterona y estrógeno en excretas de venado cola blanca (Odocoileus virginianus) como método para determinar el sexo. Revista Mexicana de Biodiversidad 87:180-186.

Loudon, A. S. y D. Curlewis (1988). Cycles of antler and testicular growth in an aseasonal tropical deer (Axis axis). Journal of reproduction y fertility 83:729-738.

MacQueen, J. B. (1967). Some methods for classification and analysis of multivariate observations. Proceedings of 5th Berkeley Symposium on Mathematical Statistics and Probability. University of California Press. Pp:281-297

Mandujano, S. (2011). Consideraciones para el manejo el manejo del venado cola blanca en UMA extensivas en bosques tropicales in Temas sobre conservación de vertebrados silvestres en México (Sánchez, O., P. Zamorano, E. Peters y H. Moya, H. eds.) SEMARNAT, México D.F., México. Pp:249-275.

Márquez, W. y J. Márquez (2009). Municipios con mayor biodiversidad en Veracruz. Foresta Veracruzana 11:43-50.

McCoy, J. C. y S. S. Ditchkoff (2012). Patterns of fecal hormones in a fenced population of white-tailed deer. Wildlife Society Bulletin 36:641-646. 
McGarigal, K., S. Cushman y S. Stafford. (2000). Multivariate statistics for wildlife and ecology research. Springer-Verlag New York, Inc. New York, E.U.A. Pp: 283.

McMillin, J. M., U. S. Seal, K. D. Keenlyne, A. W. Erickson y J. E. Jones (1974). Annual testosterone rhythm in the adult white-tailed deer (Odocoileus virginianus borealis). Endocrinology 94:1034-1040.

Miller, K. V. y L. Marchinton (1995). Quality Whitetails: The Why and How of Quality Deer Management. Stackpole Books. Mechanicsburg, E.U.A. 322 p.

Millspaugh, J. J. y B. E. Washburn (2004). Use of fecal glucocorticoid metabolite measures in conservation biology research: considerations for application and interpretation. General and Comparative Endocrinology 138:189-199.

Munro, C. y G. Stabenfeldt. (1984). Development of a microtiter plate enzyme immunoassay for the determination of progesterone. Journal of Endocrinology 101:41-49.

Miriarchi, B.E., B. E. Howland, R. E. Scanlon, R. L. Kirkpatrick y L. M. Sanford (1978). Seasonal variation in plasma LH, FSH, prolactin, and testosterone concentrations in adult male white-tailed deer. Canadian Journal of Zoology 56:121-172.

Newbolt, C. H., P. K. Acker, T. J. Neuman, S. I. Hoffman, S. S. Ditchkoff y T. D. Steury (2017). Factors influencing reproductive success in male white-tailed deer. Journal of Wildlife Management 81(2): 206-217.

NOAA (National Oceanic and Atmospheric Administration). 2018. Solar calculator. Earth System Research Laboratory. National Oceanic and Atmospheric Administration. Descargado el 21/05/18 desde: https: //www.esrl.noaa. gov/gmd/grad/solcalc/NOAA_Solar_Calculations_day.xls

Pelletier, F., J. Bauman y M. Festa-Bianchet (2003). Fecal testosterone in bighorn sheep (Ovis canadensis): behavioural and endocrine correlates. Canadian Journal of Zoology 81:1678-1684.

Piña, E. e I. Trejo (2014). Densidad poblacional y caracterización de hábitat del venado cola blanca en un bosque templado de Oaxaca, México. Acta Zoológica Mexicana (n.s.) 30:114134.

Rojo C., J. L. Cruz, G. Solano y R. Hernández (2007). Plan de manejo tipo de venado cola blanca en zonas templadas y tropicales de México. SEMARNAT, México. 30 p.

SEMARNAT (2016). Informe de la Situación del Medio Ambiente en México. Compendio de Estadísticas Ambientales. Indicadores Clave, de Desempeño Ambiental y de Crecimiento Verde. Edición 2015. Secretaría de Medio Ambiente y Recursos Naturales, México. Pp: 253255.

Stewart, J. L., C. F. Shipley, R. E. Ellerbrock, L. Schmidt, F. S. Lima e I. F. Canisso (2018). Physiological variations in reproductive and metabolic features of white-tailed deer (Odocoileus virginianus) bucks throughout the rutting season. Theriogenology 114: 308-316. 
Valdespino, C. y L. E. Martínez-Romero (2014). Determinación del ciclo reproductivo a partir de grupos fecales. in Monitoreo y manejo del venado cola blanca: Conceptos y métodos (Gallina, S., S. Mandujano y O. Villarreal. eds.) Instituto de Ecología, A. C. - Benemérita Universidad Autónoma de Puebla. Xalapa, México. 220 p.

Vaughan, C. (1994). El futuro del manejo del venado cola blanca en América Latina in Ecología y manejo del venado cola blanca en México y Costa Rica (Vaughan, C. y M. Rodríguez. eds.) Universidad Nacional, Costa Rica. 455 p.

Vega, D., S. Gallina, M. Correa, M. Parra, N. Almaraz e I. Chairez (2020). Faecal cortisol, testosterone and estradiol in white-tailed deer feces from Wildlife Management and Conservation Units in Durango, Mexico. Bio Ciencias 7 e714 https://doi.org/10.15741/revbio.07.e714

Villarreal, J. G. (1999). Venado cola blanca. Manejo y aprovechamiento cinegético. Unión Ganadera Regional de Nuevo León. Monterrey, México. 401 p.

Villarreal, J. G. (2013). Ganadería diversificada: importancia ecológica, cinegética y económica de los venados cola blanca mexicanos. SAGARPA. Monterrey, México. 237p.

Vishnu-Savanth, V. y P. C. Saseendran (2012). Impact of testosterone on the antler stages in sambar deer (Rusa unicolor) stags. Tamilnadu Journal of Veterinary y Animal Sciences 8:279285.

Waber, K., J. Spencer y P. M. Dolman (2013). Achieving landscape-scale deer management for biodiversity conservation: The need to consider sources and sinks. Journal of Wildlife Management 77:726-736.

Weerasekera, D. S., S. J. Perera, D. K. K. Nanayakkara, H. M. S. S. Herath, A. N. L. Rathnasekara y K. B. Ranawana (2020). The antler cycle and fecal testosterone of male sambar deer Rusa unicolor unicolor at the Horton Plains National Park in Sri Lanka. BioMed Research International. Article ID 6903407, 7 pages. Descargado desde: https://doi.org/10.1155/2020/6903407

XLSTAT (2017). XLSTAT software. Xlstat for Windows. Copyright 2017 Addinsoft. URL: https://www.xlstat.com/es/ (Consulta: 29 de agosto de 2018).

Yang, Y. Y., T. Borch, R. B. Young, L. D. Goodridge, and J. G. Davis (2010). Degradation kinetics of testosterone by manure-borne bacteria: influence of temperature, $\mathrm{pH}$, glucose amendments, and dissolved oxygen. J. Environ. Qual. 39:1153-1160. 